\title{
'BEING ALONE IS MORE PAINFUL THAN GETTING HURT': THE MODERATING ROLE OF WORKPLACE LONELINESS IN THE ASSOCIATION BETWEEN WORKPLACE OSTRACISM AND JOB PERFORMANCE
}

Uslu, 0.

Osman Uslu / Sakarya University, Faculty of Management, Esentepe Campus, Turkey. Email: ouslu@sakarya.edu.tr

\begin{abstract}
The consequences of both workplace ostracism and workplace loneliness may include negative impacts on employee performance, yet few studies have focused on the interactions between these three constructs. In this context, both current and future studies may make prospective contributions. The goals of this paper are to investigate the associations between these variables and to determine whether workplace loneliness has any moderating effect on the possible association between workplace ostracism and job performance. A quantitative study was designed, and 349 employees in different sectors were included in the research. Findings indicated that workplace ostracism was positively associated with workplace loneliness, while it was negatively associated with job performance. A negative association between workplace loneliness and job performance was also estimated. Neither workplace loneliness as a whole nor social companionship had any moderating effects on the workplace ostracism-job performance association. On the other hand, emotional deprivation was found to have a moderating effect on this relationship. The findings prove that in combination, emotional loneliness and ostracism cause greater damage to employee psychology and, therefore, more greatly reduce job performance.

Implications for the Central European audience: The study demonstrates that among the aspects of workplace loneliness, the emotional component is more critical to employee psychology and, therefore, job performance. Determining the factors that cause emotional loneliness in the workplace and taking precautions against them seem to be significant to the achievement of organisational goals.
\end{abstract}

Keywords: workplace ostracism; workplace loneliness; job performance JEL Classification: M12, M50

\section{Introduction}

A great number of factors affect the behaviours of individuals in organisational environments as social structures. The primary purposes of organisational behaviour, organisational psychology and behavioural sciences are to determine the factors underlying individual behaviours, to consolidate the behaviours that contribute to the production process and to improve work and social life as much as possible. Numerous studies have been conducted 
in the field to these ends. Many studies have touched on positive organisational outcomes such as job performance, job satisfaction, organisational citizenship behaviour, organisational identification and organisational trust - and emphasised on the criticality of such outcomes in the workplace. Besides these, however, it is also possible to observe undesirable behaviours in organisations. The dark side of organisational life is also a crucial issue to consider for the purposes of the field. Investigating undesired, anti-production or nonwork behaviours and identifying the factors that affect those behaviours are critical to preventing potential issues in organisational environments. Stress, burnout, organisational sabotage, gossip, envy and unfavourable conflict are just a few of them. The other two behaviours forming the dark side of organisational life, and having been recently mentioned in the field, are workplace ostracism and workplace loneliness.

Workplace ostracism is defined as one individual or group ignoring another individual or group (Williams, 2007) and neglecting to communicate with them (Robinson et al., 2013). This is considered to be an undesirable behaviour in organisations (Hitlan \& Noel, 2009). Ostracised individuals are damaged both physically and psychologically (Hitlan et al., 2006a), and trust and communication decrease significantly in the work environment. In addition to workplace ostracism's inhibitive impact on communication, trust and solidarity environments, it may also trigger undesired behaviours and attitudes such as stress, conflict, intention to leave and reduced job satisfaction (Leary et al., 2006). Another form of undesired behaviour in organisations - and one that is often confused with workplace ostracism in the literature - is workplace loneliness.

Workplace loneliness, as one of the possible consequences of workplace ostracism, is related to dissatisfaction that arises when an individual's relations with their environment are lower than the expected level (Gierveld, 1988). Workplace loneliness may cause stress, indecision, hopelessness, impatience and self-isolation in individuals (Adamson \& Axmith, 2003). The consequences of both workplace ostracism and workplace loneliness include potentially negative impacts on employee performance, and in this context, both current and future studies on the relevant variables seem to offer significant prospective contributions to the field and its practitioners. Hence, the impact of the dark side of the workplace on employee psychology (and, therefore, employee performance) was the primary focus of this study. Several research questions were created accordingly to better understand the nature of this dark side from the perspective of workplace ostracism and loneliness: What associations are present among workplace ostracism, workplace loneliness and job performance? What type of loneliness has a greater impact on job performance? Do workplace loneliness and its dimensions play any moderating role in the possible association between workplace ostracism and job performance?

In the literature, studies investigating the interactions between workplace ostracism, workplace loneliness and employee performance have been limited thus far. Indeed, these few studies have emphasised the need for further attention to the subject (Al-Atwi, 2017; Fiset et al., 2017; Liu \& Xia, 2016; Wesselmann et al., 2012). The goal of this study is to investigate the associations between workplace ostracism, workplace loneliness and job performance. Given the consequences of workplace loneliness for employees, one can also expect workplace loneliness to have a possible moderating effect. In this context, another goal of the current study is to question whether workplace loneliness and its dimensions have any moderating effects on the possible association between workplace ostracism and job 
performance. To the best of the author's knowledge, no study investigating this moderating effect has yet been conducted. Hence, this study is expected to contribute to the relevant field by exploring this unknown.

\section{Conceptual Framework and Hypotheses}

\subsection{Workplace Ostracism}

Ostracism is among a set of issues that have recently returned to the spotlight in the field of organisational behaviour. Strikingly, social ostracism is the basis of ostracism, which began to attract attention, particularly after the 2000s (Ferris et al., 2008; Hitlan et al., 2006a). Ostracised individuals tend to be homeless people, prisoners, members of dysfunctional families, criminals, elderly people or drug addicts - individuals (or members of groups) who are separated from normal people in certain respects (Levitas, 2005). Although ostracism is itself a social problem, the behavioural patterns of ostracised individuals and groups provoke the emergence of new problems. In this sense, ostracism seems to be a very sensitive subject in the behavioural sciences. The situation of ostracism can be encountered in all environments, including those of social life and work life. The problems caused by ostracism, especially in the organisational environment, point to the dark side of organisations, and they represent points of interest in the field, much like dark personality traits. Although much literature would seem to present workplace ostracism as closely related to discrimination, workplace loneliness, rejection and stigmatisation, it has been emphasised that workplace ostracism differs essentially from these other concepts (Aquino \& Thau, 2009; Robinson et al., 2013). According to Williams (2007), workplace ostracism occurs when individuals and groups ignore and exclude other individuals and groups. Robinson et al. (2013) explain workplace ostracism as the negligent failure of individuals or groups to communicate with others, even if the social relationship between parties is positive. Ostracism can take the forms of failing to establish eye contact, neglecting to greet the ostracised individual, or otherwise rejecting and ignoring them. Similarly, cases such as not inviting someone to meal or coffee breaks, acting as though they are absent in the current environment, or remaining silent (Williams, 2007) can be considered ostracism. Although Sommer et al. (2001) define ostracism as an intentional act of neglect, ostracism does is not always by design. Sometimes, unconscious ostracism can occur, as when a group in the workplace forgets to invite an individual on their way to lunch. Indeed, this unconscious ostracism is more common in work-life than is conscious ostracism (Al-Atwi, 2017).

Sanderson (2017) identifies two sides to the ostracism relationship: perpetrator and victim. Sanderson further claims that power relations between these sides play an active role. Concepts of the ostracised individual as 'target' or 'victim' are also used by other sources (Fiset et al., 2017). Liu and Xia (2016) argue that because ostracism is based on subjective perception, the situation perceived as ostracising by one individual may not be so perceived by another. Taken together, the definitions of ostracism often portray it as a form of behaviour that is detrimental to the organisation and its members. Above all, ostracism can cause longterm and recurring unhappiness (Lustenberger \& Jagacinski, 2010). Ferris et al. (2008) state that ostracism engenders a sense of social pain (Robinson et al., 2013), resulting in many negative consequences. When a person is ignored or excluded, their basic needs - such as belonging, control, self-esteem and being meaningful - become threatened (Williams et al., 2003). When these basic needs are damaged, stress, anxiety, depression (Leary et al., 
2006), and low self-esteem (Ferris et al., 2015) may manifest. Employees exposed to workplace ostracism exhibit involuntary psychological reactions such as high blood pressure, emotional exhaustion, mental depression and low job satisfaction (Ferris et al., 2008). Ostracised employees cannot engage in social interactions with co-workers (Ferris et al., 2008), which may result in weakened psychological health, increased mental fatigue or strengthened intention to quit (Ferris et al., 2008; Hitlan et al., 2006a; Wu et al., 2012). Ostracised individuals also face a lack of information in the organisational environment (Jones et al., 2009), and their contributions to work decrease (O'Reilly \& Robinson, 2009). Ostracism negatively affects environmental trust, which strengthens interpersonal relationships (Sanderson, 2017). Although a body of literature on ostracism in the workplace is developing, it is emphasised that the small degree of attention it has received (Wu et al., $2011 ; \mathrm{Xu}, 2012$ ) is not commensurate with the commonality of ostracism in work-life (Ferris et al., 2008). The majority of studies on workplace ostracism show that it is negatively correlated with behaviours that are desirable to organisations and positively correlated with undesired ones. More precisely, workplace ostracism has been found to be negatively correlated with job performance (Wu et al., 2011) and positively associated with antiproduction work behaviours (Hitlan \& Noel, 2009). Ostracism is an important stress factor (Williams, 2007) and also affects many variables in organisational environments. Prior research shows that workplace ostracism negatively affects organisational-citizenship behaviour (Fiset et al., 2017), person-organisation fit (Chung, 2015), organisational commitment (Hitlan et al., 2006b), work engagement, satisfaction with one's supervisor and co-workers (Hitlan et al., 2006a) and helping behaviours (Balliet \& Ferris, 2013; Mok \& De Cremer, 2016). On the other hand, workplace ostracism is positively associated with workfamily conflict (Liu et al., 2013), perceived racism and discrimination (Wirth \& Williams, 2009), social anxiety (Zadro et al., 2006), organisational silence (Gkorezis et al., 2016), intention to quit (Renn et al., 2013) and non-pro-social behaviours (Balliet \& Ferris, 2013; Jones et al., 2009).

\subsection{Workplace Loneliness}

Loneliness, described as an individual's feeling of being distant or removed from others (Rook, 1984), reflects a negative psychological mood. In the literature, it is noted that loneliness and workplace loneliness differ from each other. Workplace loneliness is expressed as an emotional state that occurs when the quality of interactions and communication in an organisational environment is lower than desired, and it is mostly defined as being left alone and isolated by the lonely individual's social environment (e.g. colleagues or managers) (Wright et al., 2006). The literature mentions two forms of loneliness: positive and negative. While positive loneliness refers to the voluntary movement of individuals away from their problems, negative loneliness is related to dissatisfaction that results from the inferiority, compared with expectations, of individuals' relationships with their environment (Gierveld, 1988). At this point, the 1982 assertion of Perlman \& Peplau - that the loneliness experienced by individuals means something other than being alone - seems quite meaningful. In this context, it can be argued that workplace loneliness mostly resembles negative loneliness due to its relationship with ostracism (Wright et al., 2006). Ostracism and loneliness attract attention as two topics that are frequently confused with each other in the literature. In truth, loneliness is just one of ostracism's possible outcomes. On the other hand, ostracism is not a necessary ingredient of loneliness. Various factors can cause workplace loneliness. In general, these appear to be classified as individual, situational, cultural or 
organisational factors (Levin \& Stokes, 1986; Perlman \& Peplau, 1982; Wright, 2005, 2015). According to Wright (2015), loneliness is a result of inadequate interpersonal relationships. Unwanted loneliness, isolation or lack of social support increase the strength of individuals' feelings of loneliness (Wright et al., 2006).

Previous studies have shown close relationships between loneliness and individuals' personality traits. According to the research, low self-esteem (Jones et al., 1981), shyness (Jones et al., 1981), self-consciousness (Jones et al., 1981), introversion and low initiative (Russell et al., 1980) are all associated with loneliness. Demographic and situational factors, in addition to individual ones, have been found to be associated with loneliness. In their study of deaf workers, Steinberg et al. (1999) concluded that communication barriers create a sense of loneliness and thus negatively affect performance. Furthermore, one study found that community spirit, non-work support, support from supervisors, and support from colleagues all negatively affect workplace loneliness, whereas a climate of fear positively affects workplace loneliness (Wright, 2005). Similarly, the study of Savikko et al. (2005) found that demographic and situational factors such as age, low economic status, being a widow or widower, otherwise losing a spouse, illness, and lack of friends had impacts on loneliness. Although personality and social behaviour are most commonly pointed to as the causes of workplace loneliness, environmental and organisational factors are also important antecedents. Even though workplace loneliness is an individual variable, it is ultimately a product of its organisational context (Wright, 2015). In particular, the roots of work-based loneliness seem to include a wide range of factors, such as a negative social or emotional environment, poor job design, inadequate management practices, and even departmental change, job rotation or promotion (Wright, 2005).

Workplace loneliness is generally examined through two dimensions, which are emotional deprivation and social companionship (Weiss, 1973; Wright et al., 2006). Emotional deprivation is the disappearance of individuals' emotional attachments to others; it also refers to the die-out, as a result of death or divorce, of relationships that can be described as important in human life (Weiss, 1973; Wright et al., 2006). Employees who feel emotionally deprived avoid sharing their feelings and thoughts with others, isolate themselves from their colleagues and think that no one will understand them (Wright, 2005). The social companionship dimension is related to employees' absences in workplace social networks, as well as to avoiding organisation social gatherings such as parties, dinners, funerals or birthdays (Weiss, 1973). In cases where social companionship is lacking - which can also be expressed as social loneliness or a lack of a social network (Weiss, 1973; Wright et al., 2006) - individuals may not see themselves as belonging to a group or social environment. Such a case can be understood as employees' exclusion and isolation from everyone else.

At both the individual and organisational levels, workplace loneliness seems to have mostly negative consequences. In the individual, feeling of loneliness negatively affect psychological well-being (Gumbert \& Boyd, 1984); in the work context, it causes employees to feel anxious and empty (Gierveld, 1988; Peplau \& Perlman, 1982). Furthermore, workplace loneliness may also cause stress, impatience, indecision, hopelessness and self-isolation in individuals (Adamson \& Axmith, 2003). Rokach (2004) emphasises that lonely individuals experience depleted energy and engage in negative actions; other research reveals that employees who experience loneliness in the workplace have lower levels of job satisfaction and organisational commitment (Wright, 2005). Workplace loneliness is positively correlated with 
employee intention to quit (Kaymaz et al., 2014), while it has negative effects on productivity, motivation and performance (Özçelik \& Barsade, 2011). That workplace loneliness affects organisational climate and damages corporate culture (Gumbert \& Boyd, 1984) reveals that it is a significant issue - one connected to entire organisations.

\subsection{Hypotheses}

\section{Associations among workplace ostracism, workplace loneliness and job performance}

Among the many causes of workplace loneliness, one can be expressed as workplace ostracism (Wright et al., 2006). The conditions created in individuals by workplace ostracism - such as negative psychological mood, unwillingness, mistrust, mental fatigue, burnout and dissatisfaction (Ferris et al., 2008; Hitlan et al., 2006a; Sanderson, 2017; Wu et al., 2012) trigger the factors that cause workplace loneliness. Indeed, it seems natural for ostracised individuals to experience loneliness in response to decreasing social support from their environment (Wright et al., 2006) and threats to needs such as being meaningful and belonging to a group (Williams et al., 2003). Since workplace ostracism entails that individuals are isolated from the groups to which they belong, its results include lack of communication, inadequate interpersonal relationships (Wright et al., 2006), and it indirectly leads ostracised individuals to loneliness. Although the number of studies examining the possible effects of workplace ostracism on workplace loneliness is limited, both the findings (Jones, 1990; Leary, 1990) and implications (Sanderson, 2017; Wright et al., 2006) of these studies suggest that workplace ostracism triggers workplace loneliness. In light of the relevant literature, the hypothesis developed concerning the positive association between workplace ostracism and workplace loneliness is as follows:

$\mathrm{H} 1$ : There is a positive association between workplace ostracism and workplace loneliness.

Consideration of the possible consequences of workplace ostracism reveals that it is negatively associated with organisationally desirable behaviours and positively associated with organisationally undesirable, anti-production behaviours. Since each of these organisational outcomes is directly or indirectly related to employee performance, workplace ostracism can be thought of as a potential antecedent of job performance. The information deficiencies of ostracised individuals (Jones et al., 2009) reduce the extent of their contributions to work (O'Reilly \& Robinson, 2009); this drop contribution, of course, negatively affects employees' performance quality. Research indicates that individuals who perceive a high degree of ostracism have low job-satisfaction levels (Ferris et al., 2008) and high intention to leave their jobs (Wu et al., 2012). Conditions such as weakening psychological health (Hitlan et al., 2006a), mental fatigue, psychological unwillingness and emotional burnout (Ferris et al., 2008) are expected to negatively affect job performance. The findings of previous studies also suggest that workplace ostracism will negatively affect job performance (De Clercq et al., 2019; Feng et al., 2019; Xia et al., 2019). In light of this information, a negative correlation between workplace ostracism and employee performance is expected. Thus, the following hypothesis has been developed:

$\mathrm{H}$ 2: There is a negative association between workplace ostracism and job performance.

Workplace loneliness negatively impacts the psychology of affected individuals by way of outcomes such as decreased social support, poor communication and inadequate relationships; Gumbert and Boyd (1984) state that loneliness in the workplace negatively 
affects individuals' psychological well-being. When this occurs, individuals' levels of anxiety, stress and hopelessness all increase (Adamson \& Axmith, 2003), and employees whose energy decreases may fail to engage in behaviours expected of them by their organisation (Rokach, 2004). It seems natural for employees with a sense of loneliness, who have low job satisfaction (Wright, 2005) and high intention to leave their job (Kaymaz et al., 2014), to have inferior job performance. The findings of previous studies conducted in various sectors also reveal that workplace loneliness leads to poor performance (Akçit \& Barutçu, 2017; Deniz, 2019; Özçelik \& Barsade, 2011, 2018). Accordingly, the following hypothesis has been developed:

H3: There is a negative association between workplace loneliness and job performance.

The moderating role of workplace loneliness in the association between workplace ostracism and job performance

Considering the effects of workplace ostracism and workplace loneliness on individuals, it seems natural for both to negatively affect employees' job performance (Ferris et al., 2008; Hitlan et al., 2006a; Özçelik \& Barsade, 2018; Wu et al., 2012). However, the question of whether the performance levels of ostracised individuals also change depending on their loneliness levels remains problematic. To the best of the author's knowledge, not enough study of this problem has been reported in the existing literature. From the perspective of attachment theory (Bowlby, 1982), it is inevitable that individuals who experience loneliness simultaneously feel emotionally and socially inadequate, negatively affecting their performance levels more strongly. Ostracised individuals do not always have to experience loneliness, but on the other hand, the effects of ostracism may not be as significant as the effects of loneliness. In this context, the likely negative impact of workplace ostracism on job performance is expected to be greater when employees feel lonely. As ostracised employees' levels of workplace loneliness increase, their levels of job performance are correspondingly expected to further decrease. Therefore, a moderating effect of workplace loneliness is expected on the possible association between workplace ostracism and job performance. The hypothesis developed in this context can be expressed as follows:

$\mathrm{H} 4$ : Workplace loneliness has a moderating effect on the association between workplace ostracism and job performance.

The conceptual model of the study shown in figure 1 visualises and is derived from the above hypotheses. 


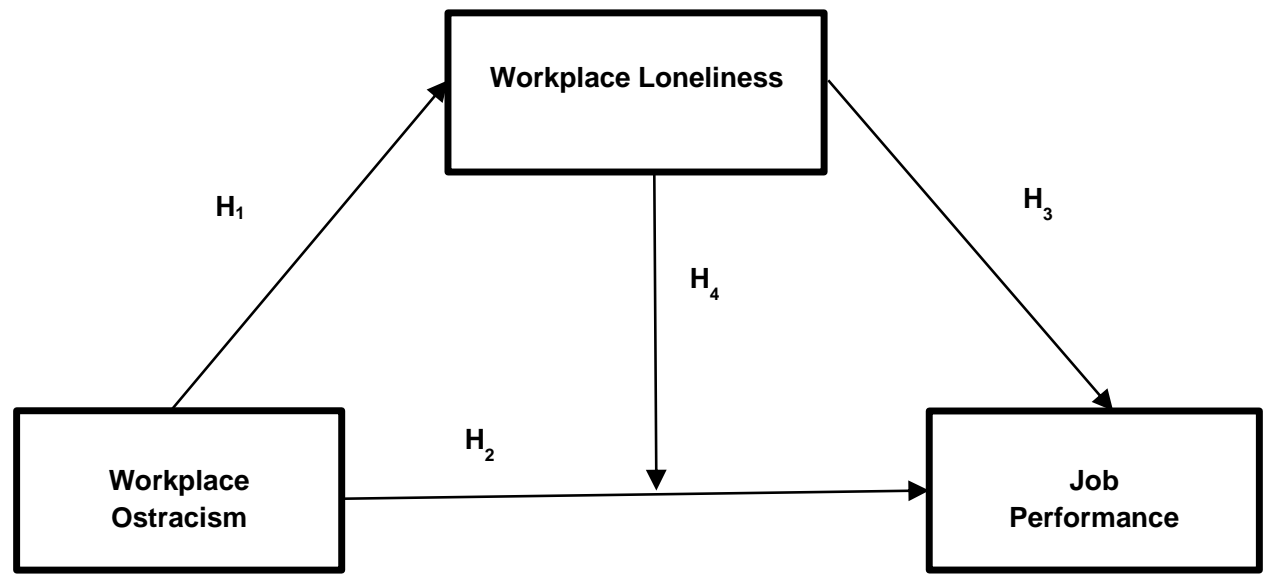

Source: authors

\section{Method}

\subsection{Design and Sample}

Quantitative research was designed with the study's objective in mind. The necessary data was gathered via an online questionnaire. Convenience sampling was adopted because there was no sectoral comparison, and the study mainly focuses on psychological aspects of workplaces. The questionnaire was developed online and sent via social media (i.e. Facebook, Instagram and LinkedIn) to the employees of organisations operating in many various sectors, including education, health, informatics and construction, in Sakarya Province, Turkey. Sakarya is a medium-sized, multicultural city located in the west of Turkey, close to İstanbul. In December 2019, the questionnaire link was sent to approximately 600 employees, who were asked to voluntarily participate in a scientific study. On that voluntary basis, 357 people participated in the questionnaire; 8 questionnaire forms were cancelled due to inconvenience. Therefore, the sample of the study consists of 349 white- and bluecollar employees. A total of 349 participants was thought sufficient to represent a city's employee population. The questionnaire consists of four sections: workplace ostracism, workplace loneliness, job performance and demographic questions.

\subsection{Measures}

\section{Workplace Ostracism Scale}

To measure workplace ostracism, a 13-item scale developed by Ferris et al. (2008) was used. The scale's translation into the local language was performed by Keklik et al. (2013). The scale consists of items such as 'Others keep away from me at work' and 'When I greet others at the workplace, it remains unrequited', which are rated on a 5-point Likert structure beginning at ' 1 - never' and ending at ' 5 - mostly'. 


\section{Workplace Loneliness Scale}

In order to measure employees' perceptions of loneliness, a 16-item workplace loneliness scale was used. The scale was developed by Wright et al. (2006) and adapted to the local language by Doğan et al. (2009). It consists of two dimensions: social companionship and emotional deprivation. This scale is of the 5-point Likert type; its structure begins at '1 strongly disagree' and ends at ' 5 - strongly agree'. It includes items such as 'There is no one at the workplace with whom to share my personal thoughts when I want to' and 'I feel emotionally distant from my colleagues'. The scale consists of two dimensions as emotional deprivation and social companionship.

\section{Job Performance Scale}

This scale, developed by Thomas and Tymon (1994), was used to measure employees' job performance. It consists of 4 items on a single dimension. The scale was adapted to the local language by Çöl (2008), and it too takes the 5-point Likert form, ranging from ' 1 - never' to ' 5 - always'. It includes items such as 'I complete my tasks on time' and 'I am sure I have reached the standards of service quality I offered'.

\section{Findings}

\subsection{Reliability and Validity Studies}

Before beginning the primary analyses, analyses of the validity and reliability of the scales were conducted. First, reliability analyses were applied to the scales, and the total item correlations of each scale were examined. During the process, the total item correlations of 2 items in the workplace ostracism scale were observed to be quite low (i.e. .113 and .287). Therefore, 2 items were excluded from the scale. No low values were found in the total item correlations of the workplace loneliness and job performance scales. The process revealed the internal consistency coefficients as $\alpha=.927$ for the workplace ostracism scale, $\alpha=.893$ for the workplace loneliness scale and ' $\alpha=.881$ for the job performance scale. Exploratory factor analysis was applied to test the validity of the scales. The analysis found that items composing the workplace ostracism and job performance scales were collected under a single factor, as in the original scales. Items forming the workplace loneliness scale were distributed over two factors, as in the original. The total explained variances were $61.181 \%$ for workplace ostracism, $61.703 \%$ for workplace loneliness and $74.223 \%$ for job performance.

These findings reveal that the scales used in this research are quite reliable and valid. Table 1 indicates the reliability coefficients and total explained variances of each scale.

\subsection{Demographic Features of Participants and Descriptive Statistics}

Table 1 also indicates in detail the scales' descriptive statistics and their statuses, depending on the participants' demographic characteristics. As shown below, the 349 participants were almost equally distributed in terms of gender and marital status. On the other hand, most participants had high school or university education levels and average monthly incomes of $€ 265-645$. In terms of age, considering that those between the ages of 18-30 made up 60\% of participants, it can be fairly said that participants were largely young. The job performance 
levels of male participants were found to be higher than those of women $(t=-2.47: p=0.01)$. Single employees were exposed to more workplace ostracism than married employees $(\mathrm{t}=$ -2.03: $p=0.04)$, and they also perceived a higher degree of loneliness $(t=-2.19: p=0.03)$. In terms of the level of education, the most ostracised employees were high school graduates $(F=2.14: p=0.07)$, and the middle-income group was exposed to workplace ostracism and perceived loneliness more than other income groups $(F=3.07: p=0.02)$. Apart from these differences, demographic characteristics did not have other significant effects on workplace ostracism, workplace loneliness or job performance.

Table 1 | Demographic features of participants, descriptive statistics and reliability coefficients

\begin{tabular}{|c|c|c|c|c|c|}
\hline $\begin{array}{l}\text { Demographic } \\
\text { Features of } \\
\text { Participants }\end{array}$ & $\mathbf{N}$ & Percent & $\begin{array}{l}\text { Workplace } \\
\text { Ostracism } \\
\text { Mean (SD) }\end{array}$ & $\begin{array}{l}\text { Workplace } \\
\text { Loneliness } \\
\text { Mean (SD) }\end{array}$ & $\begin{array}{c}\text { Job } \\
\text { Performance } \\
\text { Mean (SD) }\end{array}$ \\
\hline Gender & & & $t=1.09: p=0.28$ & $t=0.74: p=0.46$ & $t=-2.47: p=0.01$ \\
\hline Female & 180 & 51.57 & $4.74(0.57)$ & $4.04(0.70)$ & $1.65(0.66)$ \\
\hline Male & 169 & 48.43 & $4.68(0.94)$ & $3.98(0.69)$ & $1.83(0.73)$ \\
\hline Marital Status & & & $t=-2.03: p=0.04$ & $t=-2.19: p=0.03$ & $t=-0.43: p=0.66$ \\
\hline Married & 168 & 48.14 & $4.65(0.63)$ & $3.94(0.68)$ & $1.73(0.65)$ \\
\hline Single & 181 & 51.86 & $4.77(0.47)$ & $4.10(0.68)$ & $1.76(0.74)$ \\
\hline Education Level & & & $F=0.32: p=0.86$ & $F=2.14: p=0.07$ & $F=0.65: p=0.63$ \\
\hline Primary School & 42 & 12.03 & $4.73(0.55)$ & $4.01(0.68)$ & $1.78(0.76)$ \\
\hline High School & 115 & 32.95 & $4.72(0.53)$ & $4.11(0.69)$ & $1.77(0.64)$ \\
\hline Associate Degree & 80 & 22.92 & $4.75(0.56)$ & $3.84(0.70)$ & $1.75(0.74)$ \\
\hline Bachelor & 102 & 29.23 & $4.66(0.58)$ & $4.05(0.69)$ & $1.66(0.68)$ \\
\hline Graduate & 10 & 2.87 & $4.68(0.69)$ & $3.79(0.74)$ & $1.95(0.95)$ \\
\hline Monthly Income $(€)$ & & & $F=3.07: p=0.02$ & $F=3.00: p=0.02$ & $F=0.82: p=0.51$ \\
\hline 264 or below & 83 & 23.78 & $4.62(0.67)$ & $3.84(0.73)$ & $1.82(0.63)$ \\
\hline $265-645$ & 224 & 64.19 & $4.78(0.45)$ & $4.13(0.63)$ & $1.67(0.64)$ \\
\hline 646 or above & 42 & 12.03 & $4.53(0.72)$ & $3.89(0.79)$ & $1.82(0.89)$ \\
\hline Age & & & $F=0.92: p=0.46$ & $F=1.47: p=0.20$ & $F=1.46: p=0.20$ \\
\hline $18-30$ & 207 & 59.31 & $4.70(0.71)$ & $3.95(0.71)$ & $1.71(0.69)$ \\
\hline $31-40$ & 103 & 29.51 & $4.74(0.46)$ & $4.11(0.68)$ & $1.81(0.76)$ \\
\hline 41 or above & 39 & 11.18 & $4.65(0.50)$ & $4.04(0.67)$ & $1.59(0.52)$ \\
\hline Reliability & & & 0.927 & 0.893 & 0.881 \\
\hline Coefficients $(\alpha)$ & & & & & \\
\hline $\begin{array}{l}\text { Total explained } \\
\text { variance }(\%)\end{array}$ & & & 61.181 & 61.703 & 74.223 \\
\hline
\end{tabular}

Source: authors

\subsection{Hypothesis Testing}

Correlations are shown in table A (see Appendix). As is visible there, workplace ostracism, workplace loneliness (and its sub-dimensions, emotional deprivation and social companionship) and job performance were not related to any demographic variables. Only gender and job performance were found to be positively associated. Accordingly, men exhibit higher levels of job performance than women $\left(r=.13^{*} ; p<.05\right)$. Workplace ostracism was found to be positively associated with workplace loneliness $\left(r=.51^{* *} ; p<.01\right)$, emotional deprivation $\left(r=.55^{\star \star} ; p<.01\right)$ and social companionship $(r=.32 ; p<.01)$. This finding reveals that the proposed $\mathrm{H} 1$ is supported: there is a positive association between workplace ostracism and workplace loneliness.

Workplace ostracism seems to be negatively related to job performance $\left(r=-.24^{* *} ; p<.01\right)$. This low-level negative association shows that $\mathrm{H} 2$ is supported. Another finding is that the dimensions of workplace loneliness itself $\left(r=-.30^{* *} ; p<.01\right)$, emotional deprivation $(r=-$ $\left..28^{* *} ; p<.01\right)$ and social companionship $\left(r=-.23^{* *} ; p<.01\right)$ are negatively related to job performance. This also reveals that $\mathrm{H} 3$ is supported, which indicates that there is a negative association between workplace loneliness and job performance. 
Hayes's (2013) moderation analysis method was used to test H4. This technique can identify the direct effects of independent variables on dependent variables, as well as the moderating effects of third variables on this first set of effects (i.e. of independent variables on dependent variables). In this method, the mean standard deviation point of the moderator variable is grouped as the medium level, the mean standard deviation minus 1 as low the level and the mean standard deviation plus 1 as high level. Thus, the coefficient differences in the effect of the independent variable on the dependent variable at the low, medium and high levels of the moderator variable are tested. In this section, three different models were created. In the first, the moderating effect of workplace loneliness as a whole on the association between workplace ostracism and job performance was tested, while the other two models examined the moderating effects of emotional deprivation and social companionship, respectively. The findings of this analysis are shown in table 3.

Table 3 | Moderating effects of workplace loneliness, emotional deprivation and social companionship

\begin{tabular}{|c|c|c|c|c|c|}
\hline & $\beta($ se) & $t$ & $95 \% \mathrm{Cl}$ & $\Delta R^{2}$ & $F\left(d f_{1}-d f_{2}\right)$ \\
\hline WO & $-.31^{\star *}(.11)$ & -2.73 & {$[-.54 ;-.09]$} & & \\
\hline WL & $-.20^{* *}(.06)$ & -3.36 & {$[-.32 ;-.08]$} & .01 & $3.71(1-345)$ \\
\hline WO $\times W L$ & $-.15(.08)$ & -1.92 & {$[-.31 ;-.00]$} & & \\
\hline WO & $-.27^{\star \star}(.09)$ & -3.09 & {$[-.44 ;-.10]$} & & \\
\hline ED & $-.22^{* \star}(.06)$ & -3.55 & {$[-.34 ;-.10]$} & .01 & $5.19(1-345)$ \\
\hline WOXED & $-.12^{*}(.05)$ & -2.28 & {$[-.23 ;-.02]$} & & \\
\hline WO & $-.24^{\star \star}(.07)$ & -3.31 & {$[-.38 ;-.10]$} & & \\
\hline SC & $-.16^{* *}(.05)$ & -2.99 & {$[-.27 ;-.06]$} & .00 & $.99(1-345)$ \\
\hline WO $\times S C$ & $-.06(.06)$ & -1.00 & {$[-.17 ;-.06]$} & & \\
\hline
\end{tabular}

Source: authors

As seen in table 3, workplace ostracism has a direct and negative effect on job performance in the first model $(\beta=-.31 ; p<.01)$. Similarly, workplace loneliness negatively affects job performance $(\beta=-.20 ; p<.01)$. However, the interaction effect of workplace ostracism and workplace loneliness does not seem to have a significant effect on job performance $(\beta=-.15$; $p>.05)$. In the second model, similarly, workplace ostracism $(\beta=-.27 ; p<.01)$ and emotional deprivation $(\beta=-.22 ; p<.01)$ directly and negatively affect job performance. At the same time, the moderating effect of emotional deprivation is significant in the interaction of workplace ostracism and job performance $(\beta=-.12 ; p<.05)$. In the third model, although workplace ostracism $(\beta=-.24 ; p<.01)$ and social companionship $(\beta=-.16 ; p<.01)$ have direct negative effects on job performance, social companionship has no moderating effect $(\beta=-.06 ; p>.05)$. Considering these findings together, it is possible to state that $\mathrm{H} 4$, which predicts a moderating effect of workplace loneliness on the association between workplace ostracism and job performance, has been partially supported. Figure 2 shows the moderating effect of emotional deprivation on the association between workplace ostracism and job performance. As it illustrates, when emotional deprivation is low (Mean SD-1), medium (Mean $\mathrm{SD}$ ) or high (Mean $\mathrm{SD}+1$ ), the interaction coefficient of workplace ostracism and job performance becomes more strongly negative. 
Figure 2 | Moderating role of emotional deprivation on the association between workplace ostracism and job performance
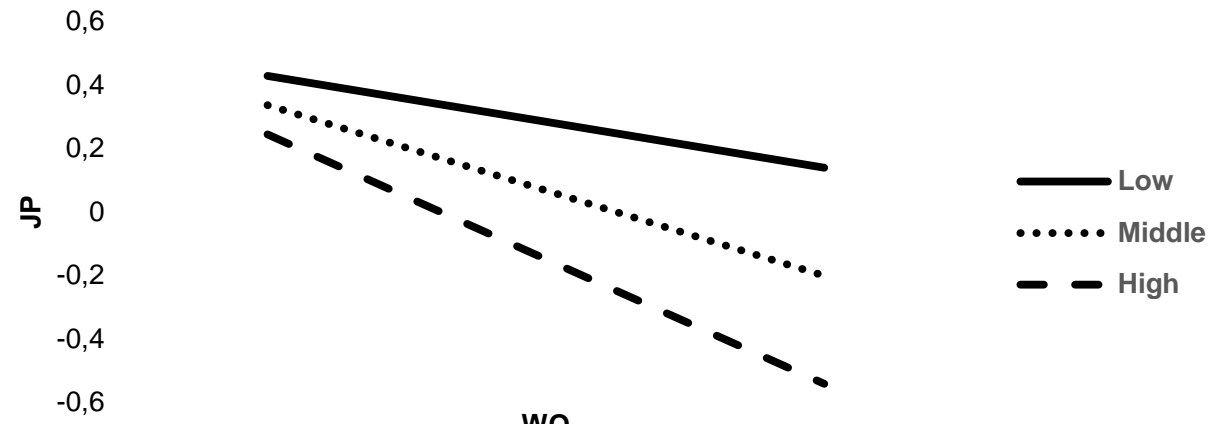

Source: authors

\section{Discussion and Conclusion}

\subsection{Workplace Ostracism, Workplace Loneliness and Job Performance}

The current study inquired as to the presence of associations among workplace ostracism, workplace loneliness and job performance, and it investigated whether workplace loneliness had a moderating effect on the association between workplace ostracism and job performance. As a result of the research, workplace ostracism has been found to be positively related to both workplace loneliness and its sub-dimensions of emotional deprivation and social companionship. This finding is consistent with those of previous studies (Leary, 1990; Li et al., 2019; Stillman et al., 2009; Wesselmann et al., 2012). Stillman et al. (2009) found that ostracism causes individuals to feel lonelier and perceive their lives as less meaningful and. Similarly, in the study of Wesselmann et al. (2012), participants who experienced loneliness reacted more negatively to ostracism than did less lonely participants. In a separate study, workplace ostracism triggered feelings of loneliness in the workplace among employees, damaging their job performance as a consequence (Li et al., 2019). Ostracised individuals naturally lose their social networks and are exposed to loneliness. A low level of trust in colleagues seems normal for individuals who feel threatened by others. In an environment with low trust, people are expected to separate themselves from others. In addition, the effects of ostracism - such as unfulfilled basic needs and the creation of certain negative psychological conditions - also harm employees emotionally and push them towards loneliness.

In the context of the current study, this explains why employees exposed to ostracism have feel emotionally deprived and perceive a lack of social companionship. At the same time, the consequences of workplace ostracism - such as high depression (DeWall et al., 2012), low levels of satisfaction with colleagues and supervisors (Hitlan et al., 2006a), high work stress (Wu et al., 2012), high anxiety (Buckner et al., 2010), psychological and physical health problems (Baumeister \& Leary, 1995), low helping behaviours (Balliet \& Ferris, 2013; Mok \& De Cremer, 2016), and low psychological well-being, organisational commitment and 
organisational citizenship behaviour (Hitlan et al., 2006b) - are likely to negatively affect employee performance. The second finding of current study is in this vein. The negative correlation between workplace ostracism and job performance is consistent with the findings previously documented in the literature (Baumeister et al., 2002; Feng et al., 2019; Ferris et al., 2015; Wu et al., 2011; Xia et al., 2019).

Another finding is a negative correlation between workplace loneliness and job performance. Employees with feelings of loneliness perform at lower levels, and as both emotional deprivation and lack of social companionship increase, the performance of the afflicted individuals decreases. Emotional deprivation was found to be interact more strongly with job performance than did lack of social companionship. This finding proves that the emotional aspect of workplace loneliness is more effective on employees than the social one. As Gumbert and Boyd (1984) state, feelings of loneliness negatively affect individuals' psychological well-being. Therefore, individuals are driven into anxiety, stress and hopelessness (Adamson \& Axmith, 2003). This explains the correlation of workplace loneliness and job performance. The findings of previous studies also point to the negative association between workplace loneliness and job performance (Akçit \& Barutçu, 2017; Deniz, 2019; Özçelik \& Barsade, 2011, 2018). Thus, findings obtained in the current study seem to in line with those of previous studies.

\subsection{Moderating Role of Workplace Loneliness}

Neither workplace loneliness nor its dimension of social companionship had significant moderating effects on the relationship between workplace ostracism and job performance. This may be a result of the fact that job performance is largely explained by workplace ostracism. On the other hand, the emotional deprivation dimension of workplace loneliness was found to have a significant moderating effect on the association between workplace ostracism and job performance; the negative interaction coefficient between workplace ostracism and job performance grows stronger as the level of emotional deprivation increases. The performance levels of employees who feel ostracised decrease even further as their emotional deprivation levels increase. This finding may be a result of the negative effects of emotional deprivation on employee psychology.

To conclude: in the context of this study, employees who are exposed to workplace ostracism feel lonelier in the workplace and exhibit poorer performance, and ostracised employees who report greater emotional deprivation perform more poorly than others.

\subsection{Implications, Limitations and Future Research}

An immediate apparently limitation is that the results of this study, which was conducted only on a Turkish sample, cannot be generalised to all cultures worldwide. Turkey is mostly characterised by a collectivist culture; in collectivist societies, matters such as cooperation, collaboration, and togetherness, in any context, closely underlie other matters. In this sense, the negative effects of ostracism and loneliness on employees might be stronger in societies characterised by much interpersonal interaction. On the other hand, Turkey has aimed to be an active participant in the EU for a long time and has therefore adopted its codes, rules and policies in many fields. This long process, combined with continuous interaction with the EU, has affected Turkish culture, which has begun to resemble EU culture. Hence, the results of this study might be partially generalised, especially to Eastern and Central European countries. 
The findings of the current study present certain implications both for academics and practitioners. First, the current study proves the significance of employees' psychological states in the workplace. Negative psychological states decrease overall firm performance as well as individual job performance. Factors affecting or potentially affecting employee psychology should be attentively considered by organisations, supervisors and policymakers. In particular, it is critical to develop practices that enable the prevention of employee ostracism and loneliness and support employees' emotional intelligence, psychological wellbeing and positive psychological capital. In this context, organisations should develop practices and trainings that strengthen collaboration, cooperation and social ties among employees. The best human-resource practices motivate employees, and work motivation increases both individual and overall organisational performance (Bienkowska \& IgnacekKuznicka, 2019; Sikyr, 2013). Social activities such as weekend meetings, parties or monthly dinners bring individuals closer together. The taking of precautions by both organisations and policymakers to prevent the negative consequences of ostracism and loneliness, as well as strict inspections of those precautionary measures, might be beneficial.

The author concedes that adopting quantitative methods alone and getting data only via surveys can be accepted as a limitation of this study. In social sciences, it may be more beneficial to use qualitative methods, especially in studies aiming to investigate employees' psychological conditions. Hence, qualitative or mixed methods may be adopted in future research, and deeper and more comprehensive and data can be obtained from employees through by way of interview techniques. In the current study, the associations of workplace ostracism and workplace loneliness were only investigated in connection with the organisational variable of job performance. However, ostracism and loneliness in the workplace may have impact not only on organisational life but also on social life. In this context, future research may examine the associations of workplace ostracism and loneliness with variables that are closely related to social life, such as broad satisfaction or psychological well-being. Ostracism damages individuals' motivating needs (such as belonging and being meaningful) and, therefore, their psychological states. Hereby, investigation of the moderating effects of the psychological conditions of individuals - such as psychological wellbeing, motivation or sense of meaning - on the association between workplace ostracism and job performance seems to be significant.

\section{References}

Adamson, B., \& Axmith, M. (2003). The CEO disconnect: Finding consistency between personal values and the demands of leadership. Ivey Business Journal, 67(5), 1-6.

Akçit, V., \& Barutçu, E. (2017). The relationship between performance and loneliness at workplace: a study on academicians. European Scientific Journal, Special Issue, 235-243. https://doi.org/10.19044/esj.2017.v13n10p\%25p.

Al-Atwi, A. A. (2017). Pragmatic impact of workplace ostracism: Toward a theoretical model. European Journal of Management and Business Economics, 26(1), 35-47. https://doi.org/10.1108/ejmbe07-2017-003.

Aquino, K., \& Thau, S. (2009). Workplace victimisation: Aggression from the target's perspective. Annual Review of Psychology, 60, 717-741. https://doi.org/10.1146/annurev.psych.60.110707.163703. 
Balliet, D., \& Ferris, D. L. (2013). Ostracism and prosocial behavior: A social dilemma perspective. Organisational Behavior and Human Decision Processes, 120(2), 298-308. https://doi.org/10.1016/j.obhdp.2012.04.004.

Baumeister, R. R., Twenge, J. M., \& Nuss, C. K. (2002). Effects of social exclusion on cognitive processes: Anticipated aloneness reduces intelligent thought. Journal of Personality and Social Psychology, 83(4), 817-827. https://doi.org/10.1037/0022-3514.83.4.817.

Baumeister, R. F., \& Leary, M. R. (1995). The need to belong: Desire for interpersonal attachments as a fundamental human motivation. Psychological Bulletin, 117(3), 497-529. https://doi.org/10.1037/0033-2909.117.3.497.

Bienkowska, A., \& Ignacek-Kuznicka, B. (2019). Influence of knowledge workers work motivation on their job performance-results of empirical research. Central European Business Review, 8(5), 54-68. https://doi.org/10.18267/j.cebr.224.

Bowlby, J. (1982). Attachment and loss: retrospect and prospect. Am J Orthopsychiatry, 52(4), 664-678. https://doi.org/10.1111/j.1939-0025.1982.tb01456.x.

Buckner, J. D., DeWall, C. N., Schmidt, N. B., \& Maner, J. K. (2010). A tale of two threats: social anxiety and attention to social threat as a function of social exclusion and non-exclusion threats. Cognitive Therapy and Research, 34(5), 449-455. https://dx.doi.org/10.1007\%2Fs10608-0099254-x.

Chung, Y. W. (2015). The role of person-organisation fit and perceived organisational support in the relationship between workplace ostracism and behavioral outcomes. Australian Journal of Management, 1-22. https://doi.org/10.1177/0312896215611190.

Çöl, G. (2008). Algılanan güçlendirmenin işgören performansı üzerindeki etkileri. [The effects of perceived empowerment on employee performance]. Doğuş Üniversitesi Dergisi, 9(1), 35-46.

De Clercq, D., Haq, I., \& Azeem, M. (2019). Workplace ostracism and job performance: roles of selfefficacy and job level. Personnel Review, 48(1), 184-203. https://dx.doi.org/10.1108/PR-022017-0039.

Gierveld, J. (1988). A Review of Loneliness: Concept and Definitions, Determinants, and Consequences. Review in Clinical Gerontology, 8, 73-80. https://doi.org/10.1017/S0959259898008090.

Deniz, S. (2019). Effect of Loneliness in the Workplace on Employees' Job Performance: A Study for Hospital Employees. International Journal of Health Services Research and Policy, 4(3), 214224. https://doi.org/10.23884/ijhsrp.2019.4.3.06.

DeWall, C. N., Gilman, R., Sharif, V., Carboni, I., \& Rice, K. G. (2012). Left out, sluggardly, and blue: Low self-control mediates the relationship between ostracism and depression. Personality and Individual Differences, 53(7), 832-837. https://doi.org/10.1016/j.paid.2012.05.025.

Doğan, T., Çetin, B., \& Sungur, M. Z. (2009). İş yaşamında yalnızlık ölçeği Türkçe formunun geçerlilik ve güvenilirlik çalışması. [Reliability and validity of Turkish version of loneliness at workplace scale]. Anadolu Psikiyatri Dergisi, 10, 271-277.

Feng, L., Li, J., Feng, T., \& Jiang, W. (2019). Workplace ostracism and job performance: Meaning at work and family support as moderators. Social Behavior and Personality: An international journal, 47(11), 1-13. https://doi.org/10.2224/sbp.8244.

Ferris, D. L., Brown, D. J., Berry, J. W., \& Lian, H. (2008). The development and validation of the workplace ostracism scale. Journal of Applied Psychology, 93(6), 1348-1366. https://doi.org/10.1037/a0012743. 
Ferris, D. L., Lian, H., Brown, D. J., \& Morrison, R. (2015). Ostracism, self-esteem, and job performance: When do we self-verify and when do we self-enhance? Academy of Management Journal, 58(1), 279-297. https://doi.org/10.5465/amj.2011.0347.

Fiset, J., Al Hajj, R., \& Vongas, J. G. (2017). Workplace ostracism seen through the lens of power. Frontiers in Psychology, 8(1528), 1-19. https://dx.doi.org/10.3389\%2Fpsyg.2017.01528.

Gkorezis, P., Panagiotou, M., \& Theodorou, M. (2016). Workplace ostracism and employee silence in nursing: the mediating role of organisational identification. Journal of Advanced Nursing, 72(10), 2381-2388. https://doi.org/10.1111/jan.12992.

Gumbert, D., \& Boyd, D. (1984). The loneliness of the small business owner. Harvard Business Review, 62(6), 33-38.

Hayes, A. F. (2013). Methodology in the social sciences. Introduction to mediation, moderation, and conditional process analysis: A regression-based approach. Guilford Press.

Hitlan, R. T., \& Noel, J. (2009). The influence of workplace exclusion and personality on counterproductive work behaviours: An interactionist perspective. European Journal of Work and Organizational Psychology, 18(4), 477-502. https://doi.org/10.1080/13594320903025028.

Hitlan, R. T., Cliffton, R. J., \& DeSoto, M. C. (2006a). Perceived exclusion in the workplace: The moderating effects of gender on work-related attitudes and psychological health. North American Journal of Psychology, 8(2), 217-235.

Hitlan, R. T., Kelly, K. M., Schepman, S., Schneider, K. T., \& Zarate, M. A. (2006b). Language exclusion and the consequences of perceived ostracism in the workplace. Group Dynamics: Theory, Research and Practice, 10(1), 56-70. https://doi.org/10.1037/1089-2699.10.1.56.

Jones, E. E., Carter-Sowell, A. R., Kelly, J. R., \& Williams, K. D. (2009). 'I'm out of the loop': Ostracism through information exclusion. Group Processes \& Intergroup Relations, 12(2), 157-174. https://doi.org/10.1177/1368430208101054.

Jones, W. H. (1990). Loneliness and social exclusion. Journal of Social and Clinical Psychology, 9(2), 214-220. https://doi.org/10.1521/jscp.1990.9.2.214.

Jones, W., Freemon, J., \& Goswick, R. (1981). The persistence of loneliness: Self and other determinants. Journal of Personality, 49(1), 27-48. https://doi.org/10.1111/j.14676494.1981.tb00844.x.

Kaymaz, K., Eroğlu, U., \& Sayılar, Y. (2014). Effect of loneliness at work on the employees' intention to leave. İş, Güç, Endüstri Iliş̧kileri ve Insan Kaynakları Dergisi, 16(1), 38-53. https://doi.org/10.4026/1303-2860.2014.0241.x.

Keklik, B., Saygın, T., \& Oral Kara, N. (2013). Akademik camianın çirkin ördek yavruları öyp'lilerde örgütsel dışlanma (ostracism) kavramının incelenmesi. [Examining organizational ostracism concept on "öyp" positions that are ugly ducklings of academic community]. 1. Örgütsel Davranış Kongresi, 351-355, Sakarya.

Leary, M. R. (1990). Responses to social exclusion: Social anxiety, jealousy, loneliness, depression, and low self-esteem. Journal of Social and Clinical Psychology, 9(2), 221-229. https://doi.org/10.1521/jscp.1990.9.2.221.

Leary, M. R., Twenge, J. M., \& Quinlivan, E. (2006). Interpersonal rejection as a determinant of anger and aggression. Personality and Social Psychology Review, 10(2), 111-132. https://doi.org/10.1207/s15327957pspr1002_2. 
Levin, I., \& Stokes, J. P. (1986). An examination of the relation individual difference variables to loneliness. Journal of Personality, 54, 717-733. https://doi.org/10.1111/j.14676494.1986.tb00422.x.

Levitas, R. (2005). The Inclusive Society? Social Exclusion and New Labour. Palgrave Macmillan, New York.

Li, Y., Mao, J. Y., \& Xu, M. (2019). Need to belong under attack: Workplace ostracism brings workplace loneliness and damaged performance. Academy of Management Proceedings, 1. https://doi.org/10.5465/ambpp.2019.16709abstract.

Liu, H., \& Xia, H. (2016). Workplace ostracism: A review and directions for future research. Journal of Human Resource and Sustainability Studies, 4, 197-201. http://dx.doi.org/10.4236/jhrss.2016.43022.

Liu, J., Kwan, H. K., Lee, C., \& Hui, C. (2013). Work-to-family spill over effects of workplace ostracism: The role of work-home segmentation preferences. Human Resource Management, 52(1), 75-94. https://doi.org/10.1002/hrm.21513.

Lustenberger, D. E., \& Jagacinski, C. M. (2010). Exploring the effects of ostracism on performance and intrinsic motivation. Human Performance, 23(4), 283-304. https://doi.org/10.1080/08959285.2010.501046.

Mok, A., \& De Cremer, D. (2016). The bonding effect of money in the workplace: priming money weakens the negative relationship between ostracism and prosocial behaviour. European Journal of Work and Organizational 286. https://doi.org/10.1080/1359432X.2015.1051038.

Psychology, 25(2), 272-

O'Reilly, J., \& Robinson, S. L. (2009). The negative impact of ostracism on Thwarted Belongingness and workplace contributions, Best paper proceedings, Academy of Management Meeting, Chicago, 1, pp. 1(7). https://doi.org/10.5465/ambpp.2009.44243707.

Özçelik, H., \& Barsade, S. (2011). Work Loneliness and Employee Performance. Academy of Management Annual Meeting Proceedings, 1(6). https://doi.org/10.5465/ambpp.2011.65869714.

Özçelik, H., \& Barsade, S. G. (2018). No employee an island: workplace loneliness and job performance. Academy of Management Journal, 61(6), 2343-2366. https://doi.org/10.5465/amj.2015.1066.

Peplau, L. A., \& Perlman, D. (1982). Perspectives on loneliness. In L. Peplau \& D. Perlman (Eds.), Loneliness: A source book of current theory, research and therapy (pp. 1-18). New York, NY: John Wiley \& Sons.

Perlman, D., \& Peplau, L. A. (1982). Theoretical approaches to loneliness. In L. Peplau \& D. Perlman (Eds.), Loneliness: A source book of current theory, research and therapy (pp. 123-134). New York, NY: John Wiley \& Sons.

Renn, R., Allen, D., \& Huning, T. (2013). The relationship of social exclusion at work with self-defeating behavior and turnover. The Journal of Social Psychology, 153(2), 229-249. https://doi.org/10.1080/00224545.2012.723642.

Robinson, L., O'Reilly, J., \& Wang, W. (2013). Invisible at work: An integrated model of workplace ostracism. Journal of Management, 39(1), 203-231. https://doi.org/10.1177/0149206312466141.

Rokach, A. (2004). Loneliness then and now: Reflections on social and emotional alienation in everyday life. Current Psychology, 23, 24-40. https://doi.org/10.1007/s12144-004-1006-1.

Rook, K. S. (1984). Promoting social bonding. American Psychologist, 39, 1389-1407. https://doi.org/10.1037/0003-066X.39.12.1389. 
Russell, D., Peplau, L. A., \& Cutrona, C. E. (1980). The revised UCLA Loneliness Scale: Concurrent and discriminant validity evidence. Journal of Personality and Social Psychology, 39(3), 472-480. https://doi.org/10.1037/0022-3514.39.3.472.

Sanderson, K. (2017). Workplace ostracism: A critical discourse of lived experience. [Unpublished doctoral dissertation]. Saint Mary's University.

Savikko, N., Routasalo, P., Tilvis, R. S., Strandberg, T. E., \& Pitkala, K. H. (2005). Predictors and subjective causes of loneliness in an aged population. Archives of Gerontology and Geriatrics, 41(3), 223-233. https://doi.org/10.1016/j.archger.2005.03.002.

Sikyr, M. (2013). Best practises in human resource management: The source of excellent performance and sustained competitiveness. Central European Business Review, 2(1), 43-48. https://doi.org/ 10.18267/j.cebr.224.

Sommer, K. L., Williams, K. D., Ciaroco, N. J., \& Baumeister, R. F. (2001). When silence speaks louder than words: explorations into the intrapsychic and interpersonal consequences of social ostracism. Basic and Applied Social Psychology, 23, 225-243. https://doi.org/10.1207/S15324834BASP2304_1.

Steinberg, A., Sullivan, V., \& Montoya, L. (1999). Loneliness and social isolation in the workforce for deaf individuals during the transition years: A preliminary investigation. Journal of Applied Rehabilitation Counselling, 30(1), 22-30. https://doi.org/10.1891/0047-2220.30.1.22.

Stillman, T. F., Baumeister, R. F., Lambert, N. M., Cresicioni, A. W., DeWall, C. N., \& Fincham, F. D. (2009). Alone and without purpose: Life loses meaning following social exclusion. Journal of Experimental Social Psychology, 45, 686-694. https://doi.org/10.1016/j.jesp.2009.03.007.

Thomas, K. W., \& Tymon, W. G. (1994). Does empowerment always work: Understanding the role of intrinsic motivation and personal interpretation. Journal of Management Systems, 6, 39-54.

Weiss, R. (1973). The experience of emotional and social isolation. Cambridge, MA: The MIT Press.

Wesselmann, E. D., Wirth, J. H., Mroczek, D. K., \& Williams, K. D. (2012). Dial a feeling: Detecting moderation of affect decline during ostracism. Personality and Individual Differences, 53, 580586. https://doi.org/10.1016/j.paid.2012.04.039.

Williams, K. D. (2007). Ostracism. Annual Review of Psychology, 58, 425-52. https://doi.org/10.1146/annurev.psych.58.110405.085641.

Williams, K. D., Case, T. I., \& Govan, C. L. (2003). Impacts of ostracism on social judgments and decisions. In J. P. Forgas \& K. D. Williams. Social judgments: Implicit and explicit processes, 5, (325-342). New York: Cambridge University Press.

Wirth, J. H., \& Williams, K. D. (2009). 'They don't like our kind': Consequences of being ostracised while possessing a group membership. Group Processes \& Intergroup Relations, 12(1), 111-127. https://doi.org/10.1177/1368430208098780.

Wright, S. L. (2005). Organisational climate, social support, and loneliness in the workplace. In N. M. Ashkanasy, W. J. Zerbe, \& C.E.J. Hartel (Series Eds.) and N. M. Ashkanasy, W. J. Zerbe, \& C.E.J. Hartel (Vol. Eds.). Research on emotion in organisations: Vol. 1. The effect of affect in organisational studies (pp. 123-142). Amsterdam, The Netherlands: Elsevier.

Wright, S. L. (2015). Coping with loneliness at work. In A. Rokach, \& A. Sha'ked (eds.), Addressing loneliness: Coping, Prevention and Clinical Interventions (123-134), Psychology Press.

Wright, S. L., Burt, C. D. B., \& Strongman, K. T. (2006). Loneliness in the workplace: Construct definition and scale development. New Zealand Journal of Psychology, 35(2), 59-68. 
Wu, L., Wei, L., \& Hui, C. (2011). Dispositional antecedents and consequences of workplace ostracism: an empirical examination. Frontiers of Business Research in China, 5(1), 23-44. https://doi.org/10.1007/s11782-011-0119-2.

Wu, L., Yim, F. H., Kwan, H. K., \& Zhang, X. (2012). Coping with workplace ostracism: The role of ingratiation and political skill in employee psychological distress. Journal of Management Studies, 49(1), 178-199. https://doi.org/10.1111/j.1467-6486.2011.01017.x.

Xia, A., Wang, B., Song, B., Zhang, W., \& Qian, J. (2019). How and when workplace ostracism influences task performance: Through the lens of conservation of resource theory. Human Resource Management Journal, 29, 353-370. https://doi.org/10.1111/1748-8583.12226.

$\mathrm{Xu}, \mathrm{H}$. (2012). How am I supposed to live without you: an investigation of antecedents and consequences of workplace ostracism? [Unpublished doctoral dissertation]. The Hong Kong Polytechnic University.

Zadro, L., Boland, C., \& Richardson, R. (2006). How long does it last? The persistence of the effects of ostracism in the socially anxious. Journal of Experimental Social Psychology, 42(5), 692-697. https://psycnet.apa.org/doi/10.1016/j.jesp.2005.10.007.

The research paper passed the review process. | Received: April 22, 2020; Revised: August 11, 2020; Accepted: August 11, 2020; Pre-published online: December 10, 2020; Published: March 19, 2021. 


\section{Appendix}

Table A | Correlations among variables

\begin{tabular}{|c|c|c|c|c|c|c|c|c|c|c|}
\hline Variables & 1 & 2 & 3 & 4 & 5 & 6 & 7 & 8 & 9 & 10 \\
\hline 1. Gender & - & & & & & & & & & \\
\hline 2. Marital Status & $.19^{\star *}$ & - & & & & & & & & \\
\hline 3. Education Level & $-.18^{\star \star}$ & $-.14^{* *}$ & - & & & & & & & \\
\hline 4. Age & $.22^{\star \star}$ & $.50^{\star *}$ & $-.13^{\star}$ & - & & & & & & \\
\hline 5. Monthly Income & $.24^{\star *}$ & $.17^{\star \star}$ & $.32^{\star \star}$ & $.27^{\star \star}$ & - & & & & & \\
\hline 6. WO & -.06 & .10 & -.04 & .01 & -.03 & - & & & & \\
\hline 7. WL & -.04 & .05 & -.04 & .09 & .05 & $.51^{\star *}$ & - & & & \\
\hline 8. ED & -.06 & .05 & -.07 & .08 & .03 & $.55^{\star *}$ & $.88^{* *}$ & - & & \\
\hline 9. SC & -.00 & .04 & -.00 & .08 & .06 & $.32^{\star *}$ & $.84^{\star *}$ & $.49^{\star *}$ & - & \\
\hline 10. JP & $.13^{*}$ & .00 & -.04 & -.04 & -.02 & $-.24^{\star *}$ & $-.30^{* *}$ & $-.28^{\star \star}$ & $-.23^{* *}$ & - \\
\hline
\end{tabular}

${ }^{*} \mathrm{p}<.05,{ }^{* *} \mathrm{p}<.01 . \mathrm{N}=349$. Gender: 1=Female, $2=$ Male. Marital status: $1=$ Married, $2=$ Single. WO=Workplace Ostracism, WL=Workplace Loneliness, ED= Emotional Deprivation, SC=Social Companionship, JP=Job Performance.

Source: author 